\title{
Cortical and Thalamic Innervation of Direct and Indirect Pathway Medium-Sized Spiny Neurons in Mouse Striatum
}

\author{
Natalie M. Doig, Jonathan Moss, and J. Paul Bolam \\ Medical Research Council Anatomical Neuropharmacology Unit, Department of Pharmacology, University of Oxford, Oxford, OX1 3TH, United Kingdom
}

The striatum receives major excitatory inputs from the cortex and thalamus that predominantly target the spines of medium-sized spiny neurons (MSNs). We aimed to determine whether there is any selectivity of these two excitatory afferents in their innervation of direct and indirect pathway MSNs. To address this, we used bacterial artificial chromosome transgenic mice, in which enhanced green fluorescent protein (EGFP) reports the presence of $\mathrm{D}_{1}$ or $\mathrm{D}_{2}$ dopamine receptor subtypes, markers of direct and indirect pathway MSNs, respectively. Excitatory afferents were identified by the selective expression of vesicular glutamate transporter type 1 (VGluT1) by corticostriatal afferents and vesicular glutamate transporter type 2 (VGluT2) by thalamostriatal afferents. A quantitative electron microscopic analysis was performed on striatal tissue from $\mathrm{D}_{1}$ and $\mathrm{D}_{2}$ mice that was double immunolabeled to reveal the EGFP and VGluT1 or VGluT2. We found that the proportion of synapses formed by terminals derived from the cortex and thalamus was similar for both direct and indirect pathway MSNs. Furthermore, qualitative analysis revealed that individual cortical or thalamic terminals form synapses with both direct and indirect pathway MSNs. Similarly, we observed a convergence of cortical and thalamic inputs onto individual MSNs of both direct and indirect pathway: individual EGFP-positive structures received input from both VGluT2-positive and VGluT2-negative terminals. These findings demonstrate that direct and indirect pathway MSNs are similarly innervated by cortical and thalamic afferents; both projections are thus likely to be critical in the control of MSNs and hence play fundamental roles in the expression of basal ganglia function.

\section{Introduction}

The striatum is the major division of the basal ganglia, and one of its primary roles is to integrate glutamatergic afferents that originate in the cortex and the thalamus (Smith and Bolam, 1990; Gerfen, 1992; Bolam et al., 2000; Smith et al., 2004; Graybiel, 2005). The predominant targets of corticostriatal and thalamostriatal projections are the dendritic spines of the medium-sized spiny neurons (MSNs), the main neuronal cell-type and principal projection neuron of the striatum (Kemp and Powell, 1971a,b; Somogyi et al., 1981; Dubé et al., 1988; Xu et al., 1989; Smith and Bolam, 1990; Xu et al., 1991; Sadikot et al., 1992; Smith et al., 1994, 2004; Bolam et al., 2000; Lacey et al., 2005; Raju et al., 2006, 2008; Moss and Bolam, 2008).

Medium-sized spiny neurons are divided into two major subpopulations based on projection targets and the expression of dopamine receptor subtypes, as well as a variety of other molecular markers. The so-called "direct pathway" neurons express the $\mathrm{D}_{1}$ dopamine receptor subtype and project directly to the output nuclei of the basal ganglia, whereas neurons of the "indirect pathway" preferentially express the $\mathrm{D}_{2}$ dopamine receptor and project

Received March 30, 2010; revised July 23, 2010; accepted Aug. 23, 2010.

This work was supported by the Medical Research Council (United Kingdom)/European Community FP7 Grant HEALTH-F2-2008-201716. N.M.D is a receipt of a Medical Research Council studentship. We thank C. R. Gerfen for helpful discussion in the initial stages of this project, M. Larsson for assistance with image analysis software, Caroline Francis, Ben Micklem, Liz Norman, and Katie Whitworth for technical support, Rob Stewart and Eleftheria Pissadaki for assistance with statistical analysis, Icnelia Huerta-0campo for assistance with immunohistochemistry, and Pablo Henny for helpful comments on this manuscript.

Correspondence should be addressed to J. P. Bolam, Medical Research Council Anatomical Neuropharmacology Unit, Department of Pharmacology, Mansfield Road, 0xford, 0X1 3TH, UK. E-mail: paul.bolam@pharm.ox.ac.uk. DOI:10.1523/JNEUROSCI.1623-10.2010

Copyright $\odot 2010$ the authors $\quad 0270-6474 / 10 / 3014610-09 \$ 15.00 / 0$ indirectly to the output nuclei via the external segment of the globus pallidus and subthalamic nucleus (Gerfen et al., 1990; Smith et al., 1998). Given that the excitatory inputs to the striatum from the cortex and thalamus are fundamental in the expression of basal ganglia function, it is critical to elucidate the pattern of innervation of the two subpopulations of MSNs. Both direct and indirect pathway MSNs have been shown to receive synaptic input from the cortex (Somogyi et al., 1981; Hersch et al., 1995; Lei et al., 2004). Light microscopic studies have suggested that the same is true for thalamostriatal afferents (Lanciego et al., 2004; Castle et al., 2005). Electron microscopic studies in primates have suggested that afferents from the centromedian nucleus (CM) of the thalamus preferentially innervate direct pathway MSNs (Sidibé and Smith, 1996). Furthermore, in vivo electrophysiological analyses have demonstrated that striatonigral neurons, at least, receive excitatory input after both cortical and thalamic stimulation (Kocsis et al., 1977; Vandermaelen and Kitai, 1980), and in vitro studies in transgenic mice (see below) have shown that individual direct and indirect pathway neurons respond to cortical and thalamic stimulation (Ding et al., 2008).

The aim of the present experiments was to define the rules of connectivity of corticostriatal and thalamostriatal afferents in relation to MSNs that give rise to the direct and indirect pathways. The development of transgenic mice that express enhanced green fluorescent protein (EGFP) under the $\mathrm{D}_{1}$ and $\mathrm{D}_{2}$ dopamine receptor promoters (Gong et al., 2003; Valjent et al., 2009) and thus identify direct and indirect pathway MSNs, respectively, together with the fact that subtypes of vesicular glutamate transporters are selective molecular markers of cortical and intralaminar thalamic afferents (VGluT1 and VGluT2, respectively) (Fremeau et al., 
2001, 2004; Herzog et al., 2001; Kaneko and Fujiyama, 2002; Fujiyama et al., 2004; Raju et al., 2006, 2008; Barroso-Chinea et al., 2008) enabled us to address this issue directly. We thus performed a quantitative analysis of the connectivity of the two glutamatergic afferents in relation to the subpopulations of MSNs.

\section{Materials and Methods}

All experimental procedures were conducted in accordance with the Animals (Scientific Procedures) Act 1986 (United Kingdom) and the Society for Neuroscience policy on the use of animals in research. Experimental procedures were performed on 28 adult bacterial artificial chromosome (BAC) transgenic mice that report subtypes of the dopamine receptor, either $\mathrm{D}_{1}$ or $\mathrm{D}_{2}$, by the presence of EGFP (28-58 g; Mutant Mouse Regional Resource Centers). Pilot and control experiments were conducted on 14 of the BAC transgenic mice to establish the optimal staining conditions, and the detailed quantitative analyses were performed on the remaining 14. Details on the methods of BAC mice production have been published and can be found on the GENSAT website (Gong et al., 2003; GENSAT, 2009).

\section{Tissue preparation}

The $\mathrm{D}_{1}$ and $\mathrm{D}_{2}$ EGFP mice were anesthetized with sodium pentobarbitone $(200 \mathrm{mg} / \mathrm{kg}$; Rhône Mérieux) and then perfused via the aorta with $\sim 30 \mathrm{ml}$ of PBS [0.01 $\mathrm{M}$ phosphate buffer (PB), pH 7.4, $0.876 \% \mathrm{NaCl}$, and $0.02 \% \mathrm{KCl}$, followed by up to $50 \mathrm{ml}$ of fixative $(0.1 \mathrm{M} \mathrm{PB}, \mathrm{pH} 7.4,3 \%$ paraformaldehyde, and $0.1 \%$ glutaraldehyde) over $\sim 15 \mathrm{~min}$. In some cases, free fixative was removed by postperfusion with PBS. The brain was removed and cut into 50-60 $\mu \mathrm{m}$ sagittal sections (1-in-6 series) using a vibrating blade microtome (VT1000S; Leica Microsystems).

\section{Immunohistochemistry}

All sections were washed three times in PBS and placed into a cryoprotectant ( $0.05 \mathrm{M} \mathrm{PB}, 25 \%$ sucrose, and $10 \%$ glycerol) for a minimum of $2 \mathrm{~h}$ before freeze-thawing. Each section was immersed in chilled isopentane and then liquid nitrogen for a few seconds, left to thaw at $\sim 25^{\circ} \mathrm{C}$ for $\sim 5$ min, and then washed three times in PBS.

The sections were then either single or double immunolabeled. All sections were immunolabeled to reveal either VGluT1 or VGluT2, as markers of cortical and thalamic terminals, respectively. Double-labeled sections were then immunolabeled to reveal the EGFP (which reports expression of either the $D_{1}$ or $D_{2}$ receptor subtypes, markers of direct or indirect pathway MSNs, respectively). Primary antibodies for the double-immunolabeling experiments were added sequentially. Normal goat serum (NGS) in PBS (Vector Laboratories) was used to block (10\% NGS) and wash (2\% NGS) sections before the addition of primary antibodies. Antibodies against VGluT1 or VGluT2, raised in rabbits (VGluT1 from MAb Technologies; VGluT2 from Synaptic Systems) were used at a dilution of 1:2000 in 2\% NGS-PBS, and the sections were incubated overnight $(\sim 14 \mathrm{~h})$, with shaking at room temperature or over 3 nights $(\sim 64 \mathrm{~h})$ at $4^{\circ} \mathrm{C}$. They were then washed once in $2 \%$ NGS-PBS, three times in PBS, and once in 1\% NGS-PBS. The sections were then incubated for $2 \mathrm{~h}$ in anti-rabbit gold-conjugated antibody $(1.4 \mathrm{~nm}$ colloidal gold; Nanoprobes) at a dilution of 1:100 in 1\% NGS-PBS. This was followed by one wash in 1\% NGS-PBS and three washes each in PBS and acetate buffer ( $0.1 \mathrm{M}$ sodium acetate 3-hydrate, $\mathrm{pH} 7.0-7.5)$ in preparation for silver intensification of the conjugated gold particles. Silver reagent ( $1 \mathrm{ml}$; HQ Silver kit; Nanoprobes) was added to each section, allowed to react for $4 \mathrm{~min}$, and washed three times each in acetate buffer and PBS and once in 2\% NGS-PBS. The sections were then incubated in a biotinylated antibody against GFP, raised in goat (BA-0702; Vector Laboratories), at a dilution of 1:500 (for $\mathrm{D}_{1}$ mouse sections) or 1:1000 (for $\mathrm{D}_{2}$ mouse sections) in $2 \%$ NGS-PBS overnight, shaking at room temperature, or over 3 nights $(\sim 64 \mathrm{~h})$ at $4^{\circ} \mathrm{C}$. Washes of once in $2 \%$ NGS-PBS and three times in PBS preceded the incubation in avidinbiotin-peroxidase complex (ABC; prepared according to the instructions of the manufacturer; Vector Laboratories) with shaking for $90 \mathrm{~min}$ at $\sim 25^{\circ} \mathrm{C}$.

After the $A B C$ incubation, the sections were washed three times in PBS, followed by three washes in $0.1 \mathrm{M} \mathrm{PB}, \mathrm{pH}$ 6.0. A peroxidase reaction using tetramethylbenzidine (TMB) as the chromogen was then performed to reveal EGFP in striatal tissue taken from $D_{1}$ and $D_{2}$ BAC mice. The TMB-tungstate method was used because of its increased sensitivity when compared with other peroxidase reactions (Bolam, 1992; Llewellyn-Smith et al., 1993). Sections were placed for $20 \mathrm{~min}$ in a preincubation solution containing the following: $80 \mathrm{ml}$ of $0.1 \mathrm{M} \mathrm{PB}, \mathrm{pH}$ 6.0, $4 \mathrm{ml}$ of ammonium paratungstate $\left(1 \%\right.$ in deionized $\left.\mathrm{H}_{2} \mathrm{O}\right), 1 \mathrm{ml}$ of TMB ( $0.2 \%$ dissolved in absolute ethanol; Sigma-Aldrich T5525), $800 \mu \mathrm{l}$ of ammonium chloride $\left(0.4 \%\right.$ in deionized $\left.\mathrm{H}_{2} \mathrm{O}\right)$, and $800 \mu \mathrm{l}$ of D-glucose $\left(20 \%\right.$ in deionized $\left.\mathrm{H}_{2} \mathrm{O}\right)$. All steps from preincubation onward were performed on ice. To perform the reaction, the preincubation solution was removed and replaced with the reaction solution, which consisted of $2 \mathrm{ml}$ of the preincubation solution (as above) plus $2 \mu \mathrm{l}$ of glucose oxidase (Sigma G6891). The reaction was allowed to continue for 2-8 min, until dark blue staining of MSN cell bodies in the striatum was clearly seen under the dissecting microscope, and was stopped with cold $0.1 \mathrm{M} \mathrm{PB}, \mathrm{pH}$ 6.0. Sections were then washed three times for $10 \mathrm{~min}$ with $0.1 \mathrm{M} \mathrm{PB}, \mathrm{pH}$ 6.0. The reaction was then stabilized using diaminobenzidine (DAB) and cobalt (II) chloride. Sections were incubated in a stabilization solution containing the following: $400 \mu \mathrm{l}$ of ammonium chloride ( $0.4 \%$ in deionized $\left.\mathrm{H}_{2} \mathrm{O}\right), 400 \mu \mathrm{l}$ of D-glucose $\left(20 \%\right.$ in deionized $\left.\mathrm{H}_{2} \mathrm{O}\right)$, $800 \mu \mathrm{l}$ of cobalt (II) chloride ( $1 \%$ in deionized $\mathrm{H}_{2} \mathrm{O}$ ), and $40 \mathrm{mg}$ of DAB dissolved in $40 \mathrm{ml}$ of $0.1 \mathrm{M} \mathrm{PB}, \mathrm{pH}$ 6.0. The stabilization solution was filtered and stored at approximately $-20^{\circ} \mathrm{C}$ before use. Sections were incubated for 10-15 min. The stabilization solution was then removed with three 10 min washes of $0.1 \mathrm{M} \mathrm{PB}, \mathrm{pH}$ 6.0. During stabilization, the blue-staining color from the reaction step changes to a darker magenta color and background staining is reduced. Sections were then washed three times in $0.1 \mathrm{M} \mathrm{PB}, \mathrm{pH} 7.4$.

The sections were then placed flat on the bottom of glass Petri dishes and postfixed in osmium tetroxide (1\% in PB; Oxkem) for $7 \mathrm{~min}$. They were then washed in $0.1 \mathrm{M} \mathrm{PB}$ and dehydrated in an ascending series of ethanol dilutions [ $15 \mathrm{~min}$ in 50\% ethanol, $35 \mathrm{~min}$ in $70 \%$ ethanol that included $1 \%$ uranyl acetate (TAAB), 15 min in $95 \%$ ethanol, and twice for $15 \mathrm{~min}$ in absolute ethanol]. After absolute ethanol, sections were washed twice in propylene oxide (Sigma) for $15 \mathrm{~min}$, lifted, using modified forceps, into resin (Durcupan ACM; Fluka) in crafted foil boats, and left overnight $(\sim 15 \mathrm{~h})$ at room temperature. The resin was then warmed to reduce its viscosity, sections were placed on microscope slides, a coverslip was applied, and the resin was cured at $65^{\circ} \mathrm{C}$ for $\sim 70 \mathrm{~h}$.

The GFP primary antibody, raised in goat, shows high affinity for wild-type, recombinant, or any variants of Aquorea victorea GFP (BA0702; Vector Laboratories). Immunoperoxidase staining of the GFP antibody showed a precise correlation with EGFP fluorescence in $\mathrm{D}_{1}$ and $\mathrm{D}_{2}$ BAC-EGFP mice. The VGluT primary antibodies were raised against rat VGluT1 and VGluT2 (amino acids 543-560 and 510-582, respectively). The distribution of immunolabeling at the light and electron microscopic levels was distinct for each primary antibody and consistent with previous observations by ourselves (Lacey et al., 2005; Moss and Bolam, 2008) and others, using the same or different antibodies (Fremeau et al., 2001; Herzog et al., 2001; Kaneko and Fujiyama, 2002; Fujiyama et al., 2004, 2006; Raju et al., 2006, 2008). No immunolabeling was observed after omission of the primary antibodies or when tissue from wild-type littermates ( $D_{1}$ BAC-EGFP negative) was processed using the anti-GFP antibody, followed by a tungstate-stabilized TMB reaction.

\section{Electron microscopic analysis}

All sections were examined in the light microscope, and areas from the dorsolateral striatum were cut from the slide, glued to the top of a resin block, and trimmed with razor blades. Serial sections, $\sim 50 \mathrm{~nm}$ thick (silver/gray), were then cut using an ultramicrotome (EM UC6; Leica Microsystems), and were collected on pioloform-coated, single-slot copper grids (Agar Scientific). The sections were then lead stained to improve contrast for electron microscopic examination. A Philips CM10 electron microscope was used to examine sections of tissue. All analyses were performed at a minimum of $5 \mu \mathrm{m}$ from the tissue-resin border (i.e., the surface of the section). The maximum distance from the tissue-resin border examined was determined by the penetration of the gold- 
conjugated antibody together with the angle at which the tissue-resin was sectioned and was therefore variable.

Tissue single labeled for VGluT1 or VGluT2 in $D_{1}$ and $D_{2}$ mice. VGluTimmunopositive synapses were systematically analyzed in one of the serial sections on an electron microscope grid. One section of the serial sections on the grid was selected at random, using randomly generated integers. At a magnification at which it is not possible to clearly visualize synapses $(1950 \times)$, an area was chosen that generally comprised the entire tissue-resin border. The magnification was then increased to $10,500 \times$, and positively labeled VGluT terminals forming asymmetric synapses were identified and digitally recorded at an indicated magnification of $25,000 \times$ (Gatan multiscan CCD camera). The criterion for an immunopositive structure was three or more silver-intensified immunogold particles (hereafter simply referred to as immunogold particles). Asymmetric synapses (Gray's type 1) were identified by the presence of presynaptic vesicle accumulation, a thick postsynaptic density, a widened synaptic cleft, and cleft material. Asymmetric synapses were identified and imaged, continuing systematically in straight lines across the section. This systematic process was continued within the same section until the entire selected area had been covered. The process was then repeated on sections on different grids until 50 VGluT-immunopositive synapses were analyzed per animal for each group: VGluT1 labeling in $\mathrm{D}_{1}$ and $\mathrm{D}_{2}$ mouse tissue, VGluT2 labeling in $\mathrm{D}_{1}$ and $\mathrm{D}_{2}$ mouse tissue $(n=3$ animals per group). This resulted in a total of 150 VGluT-positive synapses per group and an overall total of 600 asymmetric synapses. In these images, the postsynaptic target(s) of the VGluT-positive terminals were characterized. Dendritic shafts were identified by their relatively large size when compared with spines, the presence of mitochondria, and, although not essential, the protrusion of spines from their shaft. Spines were identified by their shape and relatively small size when compared with dendritic shafts, the lack of mitochondria within their cytoplasm, and, although not essential, the presence of spiny apparatus. Subsequently, any other immunopositive terminals seen within the frame were also analyzed. Any immunopositive terminals not fully within the frame were excluded. Postsynaptic structures, such as large dendrites that extended beyond the borders of the frame, were only examined within the frame. Digital images were analyzed using the publicly available software NIH ImageJ (http://rsbweb.nih.gov/ij/), and they were adjusted for contrast and brightness using Adobe Illustrator and Photoshop (version CS3; Adobe Systems).

In view of the fact that the probability of the release of glutamate at thalamostriatal synapses is less than at corticostriatal synapses in these two lines of mice (Ding et al., 2008), we analyzed quantitative aspects of the VGluT1- and VGluT 2-positive terminals in the dataset from the double-labeled tissue (see below). A total of 56 VGluT1 and 59 VGluT2 terminals forming axospinous synapses were analyzed in single sections that were systematically taken from the double-labeled tissue dataset ( $n=6$ animals per group). Cross-sectional area, length of synaptic specializations, number of vesicles, and distance of vesicles from the active zone were measured using NIH ImageJ and the NIH ImageJ plug-in PointDensitySyn (http://folk.uio.no/maxdl/software.html) (Larsson and Broman, 2005).

Tissue double labeled for VGluT1 or VGluT2 and GFP in $D_{1}$ and $D_{2}$ mice. EGFP immunopositive postsynaptic structures forming asymmetric synapses were randomly selected and followed in seven serial sections. Serial sections on one electron microscope grid were numbered from 1 to 7 at a low magnification $(58 \times)$. The analysis always began from the middle section (number 4). The tissue-resin border was located at a magnification of $5800 \times$ (at which synapses cannot be clearly visualized) and an area was then randomly selected. The magnification was then increased $(10,500 \times)$ and the tissue was scanned until the first EGFPpositive structure was identified, as indicated by the presence of TMB crystals and/or DAB staining within spines or dendrites. If the EGFPpositive structure was seen to be forming an asymmetric synapse, it was then examined and images were captured, in the seven serial sections at a magnification of $25,000 \times$, keeping the profile central within the frame. After acquisition, these images were analyzed and the presynaptic terminals forming asymmetric synapses with EGFP-positive structures were identified as being either VGluT immunopositive (three or more immu- nogold particles) or negative over the seven serial sections. It was also determined whether the structure received any other synaptic input and whether the presynaptic terminal formed more than one asymmetric synapse with the same or different postsynaptic structures. This was repeated until 50 EGFP-positive synapses had been identified and imaged in tissue from the same animal, using several grids from up to two blocks. This procedure was performed for each group $\left(D_{1}\right.$ tissue labeled for VGluT1 or VGluT2 and $\mathrm{D}_{2}$ tissue labeled for VGluT1 or VGluT2), resulting in a total of 150 EGFP-positive postsynaptic structures per group ( $n=3$ animals per group) and an overall total of 600 EGFP postsynaptic structures. To control for the fact that immunogold staining within the tissue-resin border is variable, resulting from the penetration of the immunogold secondary antibody, only sets of images (seven serial sections) containing at least one VGluT-immunopositive structure were included in the study. Any other EGFP-positive profiles receiving asymmetric synapses seen within the frame were also analyzed and counted, providing the entire presynaptic terminal was within the frame border.

\section{Statistical analysis}

All statistical calculations were performed using the statistical software $\mathrm{R}$ (version 2.10.1; R Development Core Team, 2009, Vienna, Austria) and PASW Statistics (version 18.0; SPSS, 2010, Surrey, UK). For statistical analyses, either the Fisher's exact test or the Mann-Whitney $U$ test were used, and all $p$ values described are two tailed. Differences were considered significant when $p<0.05$.

\section{Results}

\section{Postsynaptic targets of corticostriatal and thalamostriatal afferents in $D_{1}$ and $D_{2}$ transgenic mice}

The targets of cortical and thalamic innervation of the striatum, as indicated by labeling for VGluT1 or VGluT2, respectively, have been studied extensively in rat and nonhuman primate (Lacey et al., 2005; Fujiyama et al., 2006; Raju et al., 2006, 2008; Moss and Bolam, 2008). To determine whether the synaptic architecture in the $\mathrm{D}_{1}$ and $\mathrm{D}_{2}$ transgenic mice is comparable with that in other species, we analyzed tissue from the $\mathrm{D}_{1}$ and $\mathrm{D}_{2}$ BAC-EGFP mice single immunolabeled for VGluT1 or VGluT2. Light microscopic analysis revealed that the patterns of VGluT1 and VGluT2 immunogold labeling were consistent with previous reports, with intense VGluT1 labeling in the cortex and hippocampus, and VGluT2 labeling most intense in the thalamus (Fremeau et al., 2004). Electron microscopic analysis revealed that VGluT1 and VGluT2 immunogold labeling was concentrated within axons and axon terminals forming asymmetric synapses. Consistent with previous studies in rat (Lacey et al., 2005; Fujiyama et al., 2006; Raju et al., 2006; Moss and Bolam, 2008) and rhesus monkey (Raju et al., 2008) (Table 1), VGluT1-positive terminals in striatal tissue from both $\mathrm{D}_{1}$ and $\mathrm{D}_{2}$ transgenic mice formed asymmetric synapses almost exclusively with spines (98.8 and $98.2 \%$, respectively), with only a small proportion in synaptic contact with dendritic shafts (Table 1). Likewise, VGluT2-positive terminals in the $\mathrm{D}_{1}$ and $\mathrm{D}_{2}$ tissue had a similar distribution to other species, with $\sim 77-80 \%$ in contact with spines and the remainder $(\sim 21 \%)$ in contact with dendritic shafts (Table 1$)$. Statistical analysis revealed that the difference between the proportions of VGluT1- and VGluT2-positive terminals forming synapses with dendritic spines is significant ( $p<0.001$, Fisher's exact test). These data demonstrate that the pattern of glutamatergic innervation of MSNs in the striatum of the $\mathrm{D}_{1}$ and $\mathrm{D}_{2}$ BAC-EGFP transgenic mice is not markedly different from other species.

\section{Morphological characteristics of corticostriatal and thalamostriatal terminals in $D_{1}$ and $D_{2}$ transgenic mice}

A previous study revealed no differences in the cross-sectional area of VGluT1- and VGluT2-positive synaptic terminals or 
Table 1. Postsynaptic targets of individual VGluT1- and VGluT2-positive terminals in $D_{1}$ and $D_{2}$ transgenic mice compared with previous studies

\begin{tabular}{ll}
\hline Study & $\begin{array}{l}\text { Percentage } \pm \text { SEM of VGluT-positive terminals } \\
\text { forming axospinous synapses }\end{array}$ \\
\hline VGluT1 & \\
$\quad$ Mouse $D_{1}$ (present study) & $98.8 \pm 1.1(n=3)$ \\
Mouse $D_{2}$ (present study) & $98.2 \pm 1.0(n=3)$ \\
Rat (Moss and Bolam, 2008) & 95.9 \\
Rat (Lacey et al., 2005) & 98.9 \\
Rat (Raju et al., 2006) & $>90$ \\
Rat (Fujiyama et al., 2006) & 82 (patch), 81 (matrix) \\
Rhesus monkey (Raju et al., 2008) & $94-98$ \\
VGluT2 & $77.6 \pm 1.6(n=3)$ \\
Mouse $D_{1}$ (present study) & $80.1 \pm 4.8(n=3)$ \\
Mouse $D_{2}$ (present Study) & 70.5 \\
Rat (Moss and Bolam, 2008) & 71.9 \\
Rat (Lacey et al., 2005) & $80-90$ (patch), 50-60 (matrix) \\
Rat (Raju et al., 2006) & 84 (patch), 30 (matrix) \\
Rat (Fujiyama et al., 2006) & $50-65$ \\
Rhesus monkey (Raju et al., 2008) & \\
\hline
\end{tabular}

length of the synaptic membrane in the rat (Lacey et al., 2005). Similarly, no differences were observed in the diameter of VGluT-positive synaptic terminals in primate (Raju et al., 2008), although there is an indication that VGluT1-positive terminals may have a larger volume (Smith et al., 2009a). We observed no significant differences in the cross-sectional area of VGluT1- and VGluT2-positive synaptic terminals (mean \pm SD, VGluT1, $0.30 \pm 0.0495 \mu \mathrm{m}^{2}$; VGluT2, $0.30 \pm 0.0548 \mu \mathrm{m}^{2} ; p>0.05 ; n=$ 6 animals per group; Mann-Whitney $U$ test in this and subsequent comparisons) and in the length of the synaptic membrane (VGluT1, $0.18 \pm 0.05 \mu \mathrm{m}$; VGluT2, $0.16 \pm 0.01 \mu \mathrm{m} ; p>0.05$ ). Analysis of the distribution of vesicles revealed no significant differences in the number (mean \pm SD, VGluT1, $26.56 \pm 7.68$; VGluT2, $31.74 \pm 6.51 ; p>0.05$ ), density of vesicles (VGluT1, $87.77 \pm 15.65$ per $\mu \mathrm{m}^{2}$; VGluT2, $108.40 \pm 29.92$ per $\mu \mathrm{m}^{2} ; p>$ 0.05 ), mean distance from active zone (VGluT1, $241.13 \pm 52.31$ $\mathrm{nm}$; VGluT2, $254.06 \pm 43.58 \mathrm{~nm} ; p>0.05$ ), and intervesicle distance as an indicator of clustering (VGluT1, $300.48 \pm 41.07$ $\mathrm{nm}$; VGluT2, $295.35 \pm 59.44 \mathrm{~nm} ; p>0.05)$. There were also no significant differences in the morphological characteristics of terminals when pooled to reflect whether the terminal was forming a synapse with either a $D_{1}$ - or $D_{2}$-positive spine (all $p$ values $>0.05$, Mann-Whitney $U$ test). These findings indicate that there are no differences in the morphological characteristics of corticostriatal and thalamostriatal terminals and their vesicle distributions that could account for their different electrophysiological properties (Ding et al., 2008).

\section{Innervation of direct and indirect pathway MSNs by} corticostriatal and thalamostriatal terminals

In double-labeled tissue, cortical or thalamic terminals were identified by immunogold labeling for VGluT1 or VGluT2, respectively, and EGFP, which reports the expression of $\mathrm{D}_{1}$ or $\mathrm{D}_{2}$ receptors, was used to differentiate the direct and indirect pathway MSNs and was revealed by immunoperoxidase labeling. Light microscopic examination of immunolabeling for EGFP in sections from $D_{1}$ and $D_{2}$ mice showed intense labeling in the striatum and distinct labeling of the direct and indirect pathways, respectively [i.e., intense staining in the substantia nigra pars reticulata and entopeduncular nucleus $\left(D_{1}\right.$ mice $)$ and the globus pallidus externa $\left(\mathrm{D}_{2}\right.$ mice) $]$, as has been reported previously (for review, see Valjent et al., 2009). High-magnification light micros-
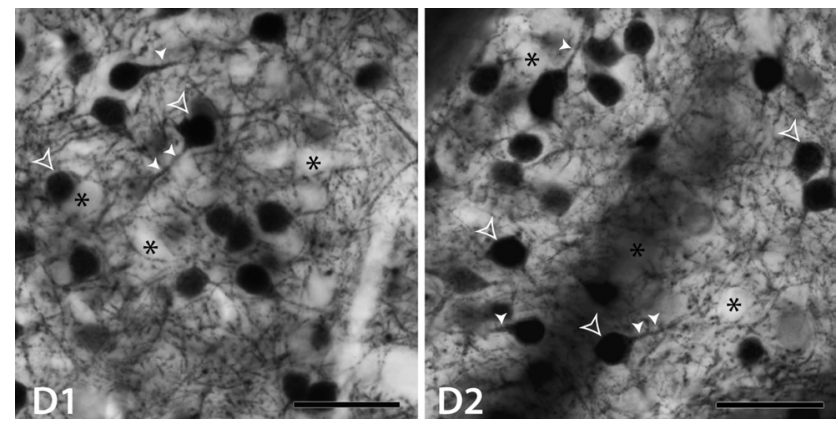

Figure 1. $D_{1}$ - and $D_{2}$-expressing MSNs in the striatum, as revealed by immunoperoxidase labeling for EGFP. Light microscopic images showing neuronal cell bodies (some indicated by open arrowheads) and dendrites (some indicated by filled arrowheads) of MSNs labeled for EGFP (immunoperoxidase reaction product; TMB-DAB) in tissue from $D_{1}$ (left) and $\mathrm{D}_{2}$ (right) BAC-EGFP mice. Note that the outlines of negative MSN cell bodies can be seen (some indicated by asterisks). Note also the dense meshwork of dendritic labeling in the neuropil. Scale bars, $50 \mu \mathrm{m}$.

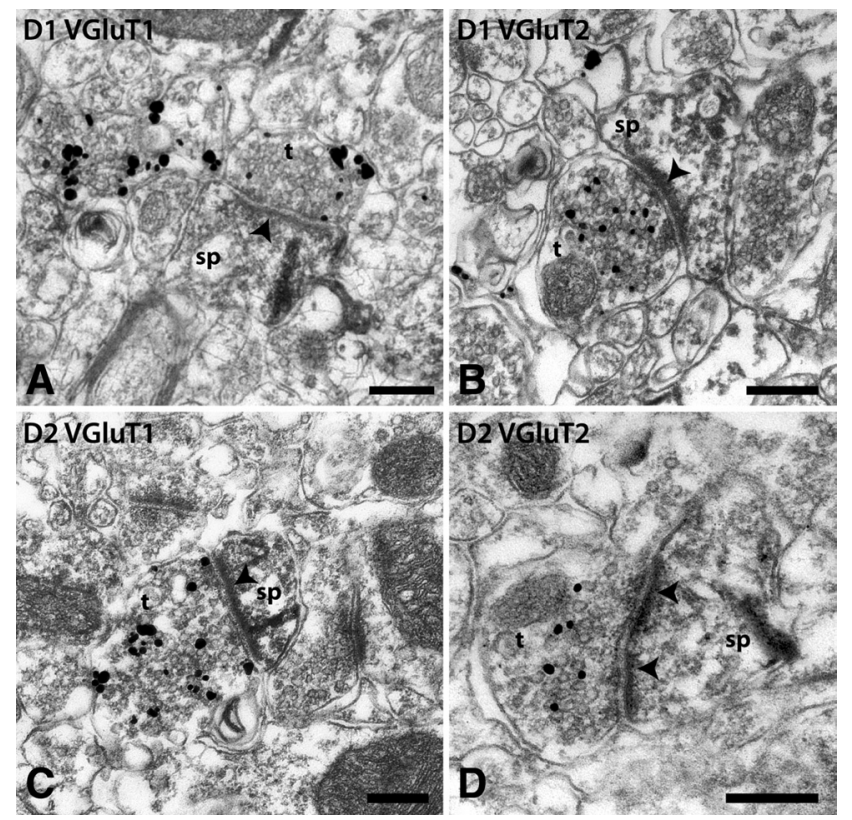

Figure 2. $\quad D_{1}$ and $D_{2}$ MSNs receive synaptic input from VGluT1-positive terminals and VGluT2-positive terminals. $A$, A spine (sp) of a $D_{1}$ MSN, identified by peroxidase immunolabeling for EGFP, receiving asymmetric synaptic input (arrowhead) from a VGluT1-positive terminal $(\mathrm{t})$, identified by immunogold labeling. Note the large crystal-like reaction product formed by TMB. $\boldsymbol{B}$, A spine (sp) of a $D_{1}$ MSN, identified by immunoperoxidase labeling for EGFP, receiving asymmetric synaptic input (arrowhead) from a VGluT2-positive terminal ( $\mathrm{t}$ ), identified by immunogold labeling. Note the floccular electron dense peroxidase reaction product. $C$, A spine (sp) of a $\mathrm{D}_{2}$ MSN identified by peroxidase labeling for EGFP receiving asymmetric synaptic input (arrowhead) from a VGluT1-positive terminal (t) identified by immunogold labeling. Note the large crystal-like reaction product formed by TMB. $D, A$ spine (sp) of $D_{2}$ MSN, identified by peroxidase labeling for EGFP, receiving asymmetric synaptic input (arrowheads) from a VGluT2positive terminal $(\mathrm{t})$ identified by immunogold labeling. Note the large crystal-like reaction product formed by TMB. Scale bars, $250 \mathrm{~nm}$.

copy revealed that labeling for EGFP was localized within MSN cell bodies, dendrites, and spine-like structures and was intermingled with unlabeled cells (Fig. 1). Electron microscopic analysis revealed that immunoperoxidase reaction product (TMB-DAB) was consistently located within MSN dendrites and dendritic spines (Fig. 2; see also Figs. 4, 5).

Initial qualitative electron microscopic analysis of the doublelabeled tissue revealed that $\mathrm{D}_{1}$ (EGFP-positive) structures receive 


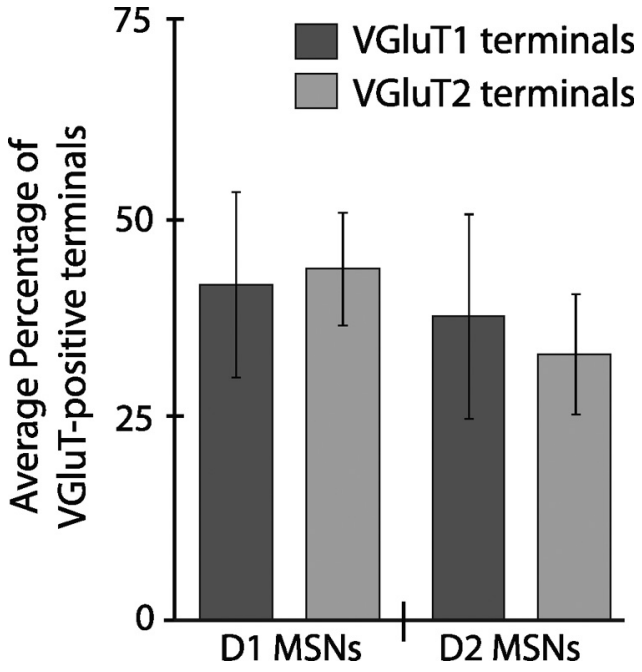

Figure 3. Quantitative analysis of the cortical and thalamic innervation of direct and indirect pathway MSNs in the striatum. A histogram showing the average percentage ( \pm SEM) of VGluTpositive terminals forming asymmetric synapses with EGFP-positive $\left(D_{1}\right.$ or $\left.D_{2}\right)$ structures out of a total of 150 synapses per set of tissue ( $D_{1}$ VGluT1, $D_{1}$ VGluT2, $D_{2}$ VGluT1, or $D_{2}$ VGluT2; $n=3$ animals per group). 0 f the $300 D_{1}$-positive structures, 63 had presynaptic structures that were VGluT1 positive and 66 that were VGluT2 positive in their respectively labeled tissue. Similarly, for $D_{2}$-positive structures, 57 had presynaptic structures that were VGluT1 positive, and 50 that were VGluT2 positive. $D_{1}$-positive or $D_{2}$-positive structures did not form significantly different numbers of synapses with VGluT1 or VGluT2 terminals ( $p>0.05$, Fisher's exact test). Additionally, neither VGluT1- nor VGluT2-positive afferents formed significantly different numbers of synapses with $D_{1}$ - or $D_{2}$-positive structures ( $p>0.05$, Fisher's exact test).

synaptic input from both VGluT1-positive terminals (Fig. 2A) and VGluT2-positive terminals (Fig. 2B). Similarly, $\mathrm{D}_{2}$ (EGFPpositive) structures receive synaptic input from both VGluT1positive terminals (Fig. 2C), and VGluT2-positive terminals (Fig. $2 D)$. This demonstrates that both cortical and thalamic afferents innervate both direct pathway MSNs and indirect pathway MSNs.

Quantitative electron microscopic analysis of the tissue double labeled for EGFP $\left(D_{1}\right.$ or $\left.D_{2}\right)$ and VGluT1 or VGluT2 was performed. Immunopositive $\left(\mathrm{D}_{1}\right.$ or $\left.\mathrm{D}_{2}\right)$ postsynaptic structures were randomly selected and analyzed, and the presynaptic terminals forming asymmetric synapses were characterized as being VGluT-positive or -negative. Thus, we were able to determine what proportion of terminals forming synaptic contact with neurons of the direct $\left(D_{1}\right)$ and indirect $\left(D_{2}\right)$ pathways were derived from the cortex (VGluT1-positive) or the thalamus (VGluT2positive) (Fig. 3). Examination of $\mathrm{D}_{1}$ tissue labeled for VGluT1 revealed that, of the 150 asymmetric synapses examined, 63 of the presynaptic terminals were positive for VGluT1, (average percentage of $42 \pm 11.5 \% ; n=3$ ) (Fig. 3). In $\mathrm{D}_{1}$ tissue labeled for VGluT2, of the 150 synapses examined, 66 of the presynaptic terminals were VGluT2 positive (average percentage of $44 \pm$ $7.02 \% ; n=3$ ) (Fig. 3). Similarly, for $\mathrm{D}_{2}$ tissue labeled for VGluT1, 57 presynaptic terminals forming synapses were positively labeled (average percentage of $38 \pm 12.7 \% ; n=3$ ) (Fig. 3). For $\mathrm{D}_{2}$ tissue labeled for VGluT2, a total of 50 of the 150 presynaptic terminals forming synapses were positive (average percentage of $33 \pm 7.42 \% ; n=3$ ) (Fig. 3). The statistical analyses revealed that the postsynaptic $\mathrm{D}_{1}$ or $\mathrm{D}_{2}$ (EGFP-positive) structures do not receive significantly different proportions of synaptic inputs from either VGluT1- or VGluT2-positive terminals ( $p=0.82$ and $p=0.47$, respectively, Fisher's exact test). Moreover, neither VGluT1- nor VGluT2-positive terminals showed any significant differences in the proportions of synapses formed with either $\mathrm{D}_{1}$ or $\mathrm{D}_{2}$ MSNs ( $p=0.56$ and $p=0.08$, respectively, Fisher's exact test).

Although the synaptic contacts made by VGluT1- and VGluT2-positive terminals with $\mathrm{D}_{1}$ - or $\mathrm{D}_{2}$-positive neurons were analyzed in separate sections, we conclude that at least a proportion of the terminals immunonegative for one of the VGluTs, in fact, expressed the other VGluT, demonstrating a convergence of cortical and thalamic input to both direct and indirect pathway neurons (see below also). However, if all VGluT-immunopositive terminals in contact with $\mathrm{D}_{1}$ or $\mathrm{D}_{2}$ neurons are considered as a whole, the total does not account for all terminals forming asymmetric synapses (15-30\% negative). Although false negatives cannot be ruled out, previous studies have shown that $\sim 20-30 \%$ of terminals forming asymmetric synapses are negative for both VGluT1 and VGluT2. This indicates that a proportion of terminals that form asymmetric synapses may not be glutamatergic or may express an hitherto unidentified vesicular glutamate transporter (Lacey et al., 2005; Raju et al., 2008; Smith et al., 2009b).

\section{Divergence and convergence of afferents derived from the cortex and thalamus in the striatum}

The fact that single VGluT-positive terminals occasionally formed more than one synapse enabled us to address, at a qualitative level, the issue of divergence of cortical and thalamic afferents with direct or indirect pathway neurons. Similarly, because EGFP-positive dendrites and spines may receive more than one synapse and we followed them in seven serial sections, we were able to examine convergence of cortical and thalamic afferent synapses formed with an individual direct or indirect pathway neuron. Of course, these data can only be considered as qualitative because of the possibility of false negatives.

We observed that individual VGluT-positive terminals formed asymmetric synapses with both an EGFP-positive spine $\left(D_{1}\right.$ or $\left.D_{2}\right)$ as well as an EGFP-negative spine (Fig. 4). Of the 63 VGluT1-positive terminals observed to form asymmetric synapses with $\mathrm{D}_{1}$-positive spines, nine also formed asymmetric synapses with $\mathrm{D}_{1}$-negative spines (Fig. $4 \mathrm{~A}$ ). Similarly, 8 of the 66 VGluT2-positive terminals that formed asymmetric synapses with $D_{1}$-positive spines also formed a synapse with a $D_{1}$-negative spine (Fig. 4B). A similar situation was observed for VGluTpositive terminals forming synapses in $\mathrm{D}_{2}$ tissue; of the 57 VGluT1-positive terminals observed to form synapses with $D_{2}$ positive spines, 9 also formed a synapse with a $\mathrm{D}_{2}$-negative spine (Fig. 4C), and 8 of the 50 VGluT2-positive terminals forming synapses with a $\mathrm{D}_{2}$-positive spine also contacted a $\mathrm{D}_{2}$-negative spine (Fig. 4D). These observations demonstrate that individual cortical and thalamic axons form divergent synaptic contact with both direct and indirect pathway MSNs.

In addition to the divergence of cortical and thalamic afferents, it was observed that an individual $D_{1}$-positive neuron received asymmetric synaptic input from both a VGluT2-positive and a VGluT2-negative terminal (Fig. 5). Similarly, this was observed for a $\mathrm{D}_{2}$-positive neuron. On these occasions, VGluT2negative synapses were formed with the heads of dendritic spines, whereas VGluT2-positive synapses were formed with the dendritic shafts from which these spines emerged. Although we cannot conclude with certainty that the VGluT2-negative terminal is of cortical origin, this indicates a convergence of excitatory inputs, from different origins, onto both direct and indirect pathway neurons. 


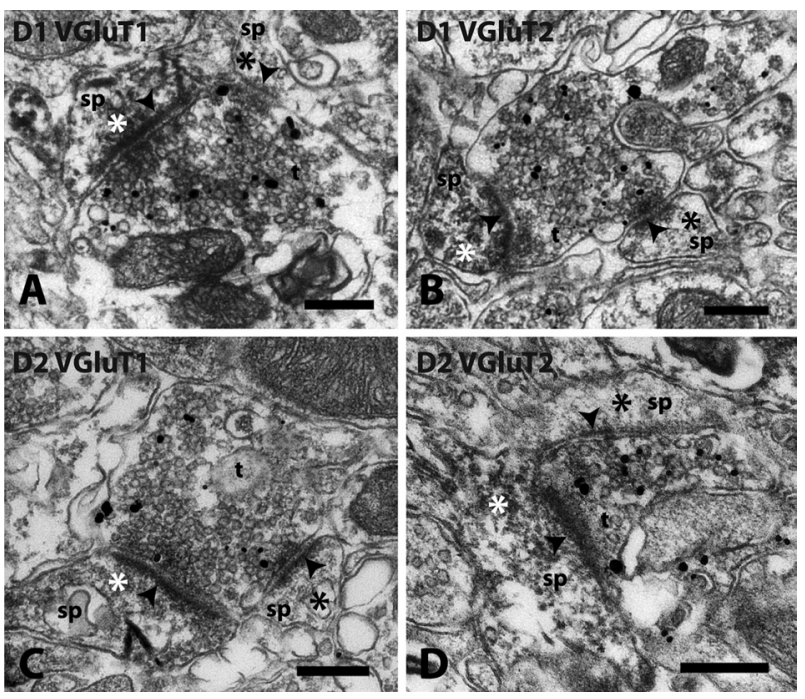

Figure 4. Divergent cortical and thalamic input to direct and indirect pathway MSN spines. $A$, A VGluT1-positive terminal (t) makes asymmetric synaptic contact (arrowheads) with a $\mathrm{D}_{1_{-}}$ positive spine (white asterisk) and a $\mathrm{D}_{1}$-negative spine (black asterisk). $\boldsymbol{B}, \mathrm{A}$ VGluT2-positive terminal ( $t$ ) makes asymmetric synaptic contact (arrowheads) with a $D_{1}$-positive spine (white asterisk) and a $\mathrm{D}_{1}$-negative spine (black asterisk). C, A VGluT1-positive terminal (t) makes asymmetric synaptic contact (arrowheads) with a $\mathrm{D}_{2}$-positive (white asterisk) and a $\mathrm{D}_{2}$-negative spine (black asterisk). D, A VGluT2-positive terminal (t) makes asymmetric synaptic contact (arrowheads) with a $\mathrm{D}_{2}$-positive spine (white asterisk) and a $\mathrm{D}_{2}$-negative spine (black asterisk). $D_{1}$ - and $D_{2}$-positive spines are identified by the presence of peroxidase reaction product; clear synaptic specializations were seen over the seven serial sections. Scale bars, $250 \mathrm{~nm}$.

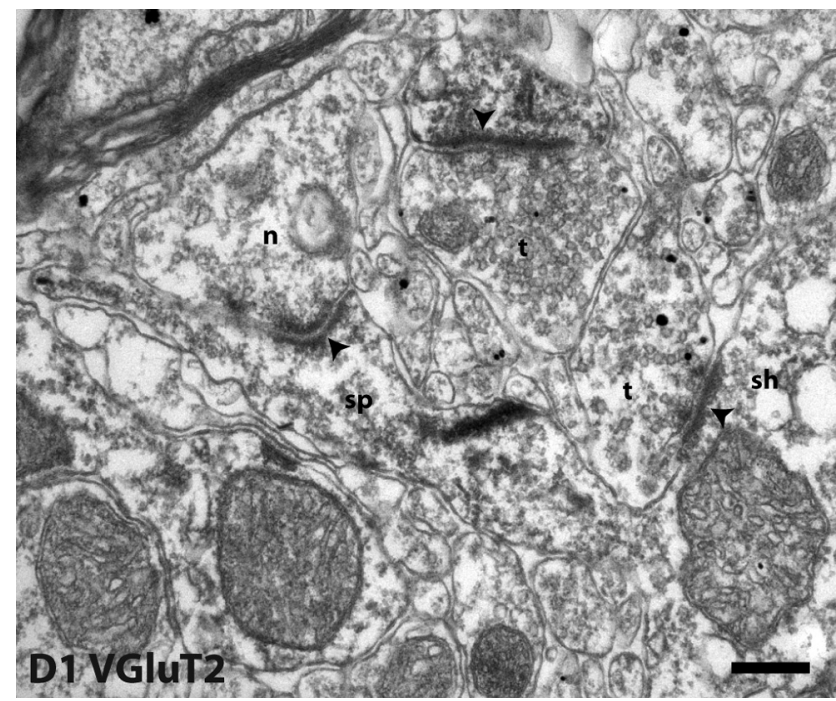

Figure 5. An individual direct pathway MSN may receive synaptic input from both thalamic (VGluT2-positive) and putative cortical (VGluT2-negative) terminals. A D shaft (sh) receives asymmetric synaptic input (arrowhead) from a VGluT2-positive terminal (t). A spine (sp) emerging from this shaft receives asymmetrical synaptic input (arrowhead) from a VGluT2-negative terminal (n). Note that at the top of the image, another VGluT2-positive terminal ( $t$ ) forms an asymmetric synapse (arrowhead) with a $\mathrm{D}_{1}$-positive spine. Scale bar, $250 \mathrm{~nm}$.

\section{Discussion}

The corticostriatal and thalamostriatal pathways, which mainly target the spines of MSNs, are critical in the expression of basal ganglia function. Furthermore, the transmission of information through the two separate, but intermingled populations of MSNs (i.e., those that give rise to the direct and indirect pathways), is one of the fundamental rules in the organization of the neuronal networks of the basal ganglia. Here we show that both corticostriatal and thalamostriatal afferents innervate MSNs that give rise to the direct pathway and MSNs that give rise to the indirect pathway. Indeed, the quantitative analyses reveal that, when considering the proportions of excitatory afferents of MSNs, the corticostriatal and thalamostriatal afferents innervate the two populations of MSNs to a similar degree. Our analyses also revealed that individual cortical and thalamic axons can innervate both direct and indirect pathway MSNs. Furthermore, the data suggest that individual direct or indirect pathway MSNs receive input from both the cortex and thalamus. Thus, a principle of basal ganglia circuitry is that the two major excitatory inputs to the basal ganglia innervate, to equal degrees, both MSNs that give rise to the direct pathways and those that give rise to the indirect pathway. Our findings are in agreement with previous electrophysiological data (Kocsis et al., 1977; Vandermaelen and Kitai, 1980; Ding et al., 2008) and reinforce the concept that subcortical inputs to the basal ganglia are as important as cortical afferents in the expression of basal ganglia function (Redgrave et al., 1999; Smith et al., 2004; McHaffie et al., 2005).

\section{Cortical and thalamic input to direct and indirect pathway MSNs}

The development of BAC transgenic mice in which EGFP reports the expression of dopamine $\mathrm{D}_{1}$ or $\mathrm{D}_{2}$ receptors and hence direct or indirect pathway MSNs has been critical in the elucidation of the properties of these subpopulations of MSNs (Gong et al., 2003; Lobo et al., 2006; Kreitzer and Malenka, 2007; Surmeier et al., 2007; Ade et al., 2008; Cepeda et al., 2008; Day et al., 2008; Gertler et al., 2008; Shen et al., 2008; Shuen et al., 2008; Janssen et al., 2009). This development, together with the discovery that VGluT1 and VGluT2 selectively label corticostriatal and thalamostriatal afferents (Fremeau et al., 2004), enabled us to directly assess and quantify the relationship between excitatory afferents and the principal neurons of the striatum. We demonstrated that both corticostriatal and thalamostriatal projections make synaptic contact both direct and indirect pathway neurons to similar degrees. The vast majority of synapses analyzed were axospinous. However, we cannot rule out the possibility of axodendritic synapses in contact with interneurons, but it should be noted that these only account for a very small proportion of the synapses analyzed. Our findings thus suggest that, despite heterogeneities in neurochemistry, morphology, and connections of the two populations of MSNs, when using molecular markers to study the projections, the corticostriatal and thalamostriatal afferents are relatively uniform in their innervation of MSNs. It is possible, however, that there is heterogeneity when considering subdivisions of the corticostriatal or thalamostriatal pathways. Indeed, previous anterograde labeling studies have indicated that subpopulations of cortical afferents show selectivity for direct or indirect pathway MSNs (Hersch et al., 1995; Lei et al., 2004), and afferents from the CM nucleus of the squirrel monkey selectively innervate neurons of the direct pathway (Sidibé and Smith, 1996). Furthermore, microstimulation of sensorimotor cortex and local disinhibition of primary motor cortex both result in upregulation of early-gene expression primarily within indirect pathway neurons (Berretta et al., 1997; Parthasarathy and Graybiel, 1997). Moreover, lesioning of the intralaminar thalamic nuclei has been shown to preferentially downregulate enkephalin mRNA, thus indicating an increased influence over the indirect pathway (Salin and Kachidian, 1998). Our study does not obviate these findings but demonstrates that, when the projections are considered as a whole, there is uniformity in the innervation of the two populations of 
MSNs by excitatory afferents, and hence both direct and indirect pathways neurons are likely to be similarly influenced by cortical and thalamic afferents.

It is critical to determine whether the homogeneity of the excitatory innervation of MSN subpopulations is reflected in the innervation of single cells, i.e., do individual direct or indirect pathway MSNs receive convergent input from both the cortex and the thalamus and, if so, what are the relative proportions of these afferents? Our electron microscopic analyses enabled us to provide qualitative data on this issue. On the basis that a terminal that is negative for, say, VGluT2 is likely to be a cortical terminal (i.e., presumably expressing VGluT1) and vice versa, we identified that individual direct pathway MSNs or indirect pathway MSNs receive input from both the cortex and thalamus. However, we should be cautious in our interpretation because a terminal immunonegative for one of the VGluTs may simply be a false negative; furthermore, it does not necessarily mean that it expresses the other VGluT examined in this study. The fact that we observed that $56-67 \%$ of terminals forming asymmetric synapses with $\mathrm{D}_{1}$ or $\mathrm{D}_{2}$ MSNs are negative for the VGluT under study implies convergence of cortical and thalamic terminals on individual neurons, because together VGluT1- or VGluT2-positive terminals account for $70-85 \%$ of asymmetric synapses in the striatum. Thus, at least some of the negative terminals will express the other VGluT. Direct analysis of this issue requires the combination of VGluT immunolabeling together with the labeling of single, identified, direct or indirect pathway MSNs. Our findings thus confirm and extend in vivo electrophysiological analyses that have revealed that individual MSNs frequently respond to both cortical and thalamic stimulation and in vitro analyses demonstrating both cortical and thalamic input to direct and indirect pathway neurons (Kocsis et al., 1977; Vandermaelen and Kitai, 1980; Ding et al., 2008; Smeal et al., 2008).

A second critical issue in the organization of the corticostriatal and thalamostriatal pathways is the divergence of cortical and thalamic afferents to the striatum. Do individual cortical or thalamic axons innervate both direct and indirect pathway MSNs? Again, our observations threw light on this issue. Individual cortical terminals were identified forming synapses with both direct pathway MSNs in the $D_{1}$ mice and presumed indirect pathway MSNs that were immunonegative for EGFP. This suggests that an individual cortical axon provides divergent input to the striatum with respect to subpopulations of MSNs, a conclusion that was also made from the analysis of the $\mathrm{D}_{2}$ mice. On the basis of similar analyses, we also conclude that individual thalamic axons provide divergent input to subpopulations of MSNs. The precise quantitative aspects of convergence and divergence remain to be established.

The classical view of the functional organization of the basal ganglia is that corticostriatal projections provide the major inputs to the basal ganglia and the role of thalamus is transmission of information back to the cortex. However, it is clear that subcortical inputs to the basal ganglia are prominent and, in evolutionary terms, preceded the cortical input (McHaffie et al., 2005). Prominent, and probably most important, of the subcortical inputs is that from the thalamus (Groenewegen and Berendse, 1994; Smith et al., 2004), principally, but not exclusively, from the intralaminar thalamic nuclei. Indeed, there are many similarities between the thalamostriatal and the corticostriatal pathways in addition to their glutamatergic phenotype. Quantitative analysis has demonstrated that the number of synapses in the striatum derived from the thalamus is of the same order of magnitude as derived from the cortex (Lacey et al., 2005; Raju et al., 2008).
Furthermore, their synaptic targets are essentially similar, in that most cortical and thalamic terminals innervate dendritic spines with a smaller proportion innervating the dendritic shafts of interneurons (Kemp and Powell, 1971b; Somogyi et al., 1981; Dubé et al., 1988; Xu et al., 1989; Smith and Bolam, 1990; Xu et al., 1991; Sadikot et al., 1992; Bennett and Bolam, 1994; Smith et al., 2004). Of course, a notable exception is the thalamostriatal pathway originating in the parafascicular nucleus, which principally innervates dendritic shafts (Raju et al., 2006; Lacey et al., 2007). The spatial relationship between thalamostriatal synapses and dopaminergic synapses is similar to the relationship between corticostriatal and dopaminergic synapses; thus, both are in a position to be modulated by released dopamine to a similar degree (Moss and Bolam, 2008). Despite differences in the response to stimulation of corticostriatal and thalamostriatal afferents (Smeal et al., 2007, 2008), which do not vary between direct and indirect pathway neurons (Ding et al., 2008), they are both excitatory and give rise to EPSPs. Any specific differences between corticostriatal and thalamostriatal projections are likely to be underscored by the particular neurons within different functional regions of the cortex or subnuclei thalamus that give rise to the projections. The thalamostriatal projection, in particular, is highly heterogeneous with respect to the somatodendritic morphology of the projecting neurons, their electrophysiological activity, synaptic targets, and patterns of innervation of the matrix and patch/striosomes (Herkenham and Pert, 1981; Deschênes et al., 1996; Smith et al., 2004; Raju et al., 2006; Lacey et al., 2007).

\section{Functional implications}

The neuronal networks that constitute the basal ganglia and the regions that are connected to the basal ganglia are organized in such a way that excitatory inputs to the striatum "select" which individual MSNs, or groups of MSNs, are depolarized from the relatively hyperpolarized resting state. With sufficient convergent excitatory input and appropriate modulatory influence of released dopamine, the selected population of MSNs, which likely constitute both direct and indirect pathway neurons, will fire action potentials, and it is this that underlies basal gangliaassociated behavior. Our findings, together with those of others, suggest that, although the thalamostriatal pathway carries different functional information from that of the corticostriatal pathway and encodes it in an heterogeneous manner (Lacey et al., 2007) that is distinct from the cortex in terms of receptor profiles, release probability, and short-term plasticity (Ding et al., 2008; Smeal et al., 2008), it is likely to be equally involved in the selection of individual or populations of MSNs of both the direct and indirect pathways.

\section{References}

Ade KK, Janssen MJ, Ortinski PI, Vicini S (2008) Differential tonic GABA conductances in striatal medium spiny neurons. J Neurosci 28:11851197.

Barroso-Chinea P, Castle M, Aymerich MS, Lanciego JL (2008) Expression of vesicular glutamate transporters 1 and 2 in the cells of origin of the rat thalamostriatal pathway. J Chem Neuroanat 35:101-107.

Bennett BD, Bolam JP (1994) Synaptic input and output of parvalbuminimmunoreactive neurons in the neostriatum of the rat. Neuroscience 62:707-719.

Berretta S, Parthasarathy HB, Graybiel AM (1997) Local release of GABAergic inhibition in the motor cortex induces immediate-early gene expression in indirect pathway neurons of the striatum. J Neurosci 17:4752-4763.

Bolam JP, ed (1992) Experimental neuroanatomy. Oxford: Oxford UP.

Bolam JP, Hanley JJ, Booth PA, Bevan MD (2000) Synaptic organisation of the basal ganglia. J Anat 196:527-542.

Castle M, Aymerich MS, Sanchez-Escobar C, Gonzalo N, Obeso JA, Lanciego 
JL (2005) Thalamic innervation of the direct and indirect basal ganglia pathways in the rat: ipsi- and contralateral projections. J Comp Neurol 483:143-153.

Cepeda C, André VM, Yamazaki I, Wu N, Kleiman-Weiner M, Levine MS (2008) Differential electrophysiological properties of dopamine D1 and D2 receptor-containing striatal medium-sized spiny neurons. Eur J Neurosci 27:671-682.

Day M, Wokosin D, Plotkin JL, Tian X, Surmeier DJ (2008) Differential excitability and modulation of striatal medium spiny neuron dendrites. J Neurosci 28:11603-11614.

Deschênes M, Bourassa J, Doan VD, Parent A (1996) A single-cell study of the axonal projections arising from the posterior intralaminar thalamic nuclei in the rat. Eur J Neurosci 8:329-343.

Ding J, Peterson JD, Surmeier DJ (2008) Corticostriatal and thalamostriatal synapses have distinctive properties. J Neurosci 28:6483-6492.

Dubé L, Smith AD, Bolam JP (1988) Identification of synaptic terminals of thalamic or cortical origin in contact with distinct medium-size spiny neurons in the rat neostriatum. J Comp Neurol 267:455-471.

Fremeau RT Jr, Troyer MD, Pahner I, Nygaard GO, Tran CH, Reimer RJ, Bellocchio EE, Fortin D, Storm-Mathisen J, Edwards RH (2001) The expression of vesicular glutamate transporters defines two classes of excitatory synapse. Neuron 31:247-260.

Fremeau RT Jr, Voglmaier S, Seal RP, Edwards RH (2004) VGLUTs define subsets of excitatory neurons and suggest novel roles for glutamate. Trends Neurosci 27:98-103.

Fujiyama F, Kuramoto E, Okamoto K, Hioki H, Furuta T, Zhou L, Nomura S, Kaneko T (2004) Presynaptic localization of an AMPA-type glutamate receptor in corticostriatal and thalamostriatal axon terminals. Eur J Neurosci 20:3322-3330.

Fujiyama F, Unzai T, Nakamura K, Nomura S, Kaneko T (2006) Difference in organization of corticostriatal and thalamostriatal synapses between patch and matrix compartments of rat neostriatum. Eur J Neurosci 24:2813-2824.

GENSAT (2009) The Gene Expression Nervous System Atlas (GENSAT) Project. National Institute of Neurological Disorders and Stroke Contracts N01NS02331 and HHSN271200723701C. Rockefeller University, New York. http://www.gensat.org/index.html.

Gerfen CR (1992) The neostriatal mosaic: multiple levels of compartmental organization in the basal ganglia. Annu Rev Neurosci 15:285-320.

Gerfen CR, Engber TM, Mahan LC, Susel Z, Chase TN, Monsma FJ Jr, Sibley DR (1990) D1 and D2 dopamine receptor-regulated gene expression of striatonigral and striatopallidal neurons. Science 250:1429-1432.

Gertler TS, Chan CS, Surmeier DJ (2008) Dichotomous anatomical properties of adult striatal medium spiny neurons. J Neurosci 28:1081410824 .

Gong S, Zheng C, Doughty ML, Losos K, Didkovsky N, Schambra UB, Nowak NJ, Joyner A, Leblanc G, Hatten ME, Heintz N (2003) A gene expression atlas of the central nervous system based on bacterial artificial chromosomes. Nature 425:917-925.

Graybiel AM (2005) The basal ganglia: learning new tricks and loving it. Curr Opin Neurobiol 15:638-644.

Groenewegen HJ, Berendse HW (1994) The specificity of the "nonspecific" midline and intralaminar thalamic nuclei. Trends Neurosci 17:52-57.

Herkenham M, Pert CB (1981) Mosaic distribution of opiate receptors, parafascicular projections and acetylcholinesterase in rat striatum. Nature 291:415-418.

Hersch SM, Ciliax BJ, Gutekunst CA, Rees HD, Heilman CJ, Yung KK, Bolam JP, Ince E, Yi H, Levey AI (1995) Electron microscopic analysis of $D_{1}$ and $\mathrm{D}_{2}$ dopamine receptor proteins in the dorsal striatum and their synaptic relationships with motor corticostriatal afferents. J Neurosci 15:52225237.

Herzog E, Bellenchi GC, Gras C, Bernard V, Ravassard P, Bedet C, Gasnier B, Giros B, El Mestikawy S (2001) The existence of a second vesicular glutamate transporter specifies subpopulations of glutamatergic neurons. J Neurosci 21:RC181(1-6).

Janssen MJ, Ade KK, Fu Z, Vicini S (2009) Dopamine modulation of GABA tonic conductance in striatal output neurons. J Neurosci 29:5116-5126.

Kaneko T, Fujiyama F (2002) Complementary distribution of vesicular glutamate transporters in the central nervous system. Neurosci Res 42:243-250.

Kemp JM, Powell TP (1971a) The site of termination of afferent fibres in the caudate nucleus. Philos Trans R Soc Lond B Biol Sci 262:413-427.
Kemp JM, Powell TP (1971b) The termination of fibres from the cerebral cortex and thalamus upon dendritic spines in the caudate nucleus: a study with the Golgi method. Philos Trans R Soc Lond B Biol Sci 262:429-439.

Kocsis JD, Sugimori M, Kitai ST (1977) Convergence of excitatory synaptic inputs to caudate spiny neurons. Brain Res 124:403-413.

Kreitzer AC, Malenka RC (2007) Endocannabinoid-mediated rescue of striatal LTD and motor deficits in Parkinson's disease models. Nature 445:643-647.

Lacey CJ, Boyes J, Gerlach O, Chen L, Magill PJ, Bolam JP (2005) GABA(B) receptors at glutamatergic synapses in the rat striatum. Neuroscience 136:1083-1095.

Lacey CJ, Bolam JP, Magill PJ (2007) Novel and distinct operational principles of intralaminar thalamic neurons and their striatal projections. J Neurosci 27:4374-4384.

Lanciego JL, Gonzalo N, Castle M, Sanchez-Escobar C, Aymerich MS, Obeso JA (2004) Thalamic innervation of striatal and subthalamic neurons projecting to the rat entopeduncular nucleus. Eur J Neurosci 19:12671277.

Larsson M, Broman J (2005) Different basal levels of CaMKII phosphorylated at Thr286/287 at nociceptive and low-threshold primary afferent synapses. Eur J Neurosci 21:2445-2458.

Lei W, Jiao Y, Del Mar N, Reiner A (2004) Evidence for differential cortical input to direct pathway versus indirect pathway striatal projection neurons in rats. J Neurosci 24:8289-8299.

Llewellyn-Smith IJ, Pilowsky P, Minson JB (1993) The tungstate-stabilized tetramethylbenzidine reaction for light and electron microscopic immunocytochemistry and for revealing biocytin-filled neurons. J Neurosci Methods 46:27-40.

Lobo MK, Karsten SL, Gray M, Geschwind DH, Yang XW (2006) FACSarray profiling of striatal projection neuron subtypes in juvenile and adult mouse brains. Nat Neurosci 9:443-452.

McHaffie JG, Stanford TR, Stein BE, Coizet V, Redgrave P (2005) Subcortical loops through the basal ganglia. Trends Neurosci 28:401-407.

Moss J, Bolam JP (2008) A dopaminergic axon lattice in the striatum and its relationship with cortical and thalamic terminals. J Neurosci 28:11221-11230.

Parthasarathy HB, Graybiel AM (1997) Cortically driven immediate-early gene expression reflects modular influence of sensorimotor cortex on identified striatal neurons in the squirrel monkey. J Neurosci 17:2477-2491.

Raju DV, Shah DJ, Wright TM, Hall RA, Smith Y (2006) Differential synaptology of VGluT2-containing thalamostriatal afferents between the patch and matrix compartments in rats. J Comp Neurol 499:231-243.

Raju DV, Ahern TH, Shah DJ, Wright TM, Standaert DG, Hall RA, Smith Y (2008) Differential synaptic plasticity of the corticostriatal and thalamostriatal systems in an MPTP-treated monkey model of parkinsonism. Eur J Neurosci 27:1647-1658.

Redgrave P, Prescott TJ, Gurney K (1999) The basal ganglia: a vertebrate solution to the selection problem? Neuroscience 89:1009-1023.

Sadikot AF, Parent A, Smith Y, Bolam JP (1992) Efferent connections of the centromedian and parafascicular thalamic nuclei in the squirrel monkey: a light and electron microscopic study of the thalamostriatal projection in relation to striatal heterogeneity. J Comp Neurol 320:228-242.

Salin P, Kachidian P (1998) Thalamo-striatal deafferentation affects preproenkephalin but not preprotachykinin gene expression in the rat striatum. Brain Res Mol Brain Res 57:257-265.

Shen W, Flajolet M, Greengard P, Surmeier DJ (2008) Dichotomous dopaminergic control of striatal synaptic plasticity. Science 321:848-851.

Shuen JA, Chen M, Gloss B, Calakos N (2008) Drdla-tdTomato BAC transgenic mice for simultaneous visualization of medium spiny neurons in the direct and indirect pathways of the basal ganglia. J Neurosci 28:26812685.

Sidibé M, Smith Y (1996) Differential synaptic innervation of striatofugal neurones projecting to the internal or external segments of the globus pallidus by thalamic afferents in the squirrel monkey. J Comp Neurol 365:445-465.

Smeal RM, Gaspar RC, Keefe KA, Wilcox KS (2007) A rat brain slice preparation for characterizing both thalamostriatal and corticostriatal afferents. J Neurosci Methods 159:224-235.

Smeal RM, Keefe KA, Wilcox KS (2008) Differences in excitatory transmission between thalamic and cortical afferents to single spiny efferent neurons of rat dorsal striatum. Eur J Neurosci 28:2041-2052. 
Smith AD, Bolam JP (1990) The neural network of the basal ganglia as revealed by the study of synaptic connections of identified neurones. Trends Neurosci 13:259-265.

Smith Y, Bennett BD, Bolam JP, Parent A, Sadikot AF (1994) Synaptic relationships between dopaminergic afferents and cortical or thalamic input in the sensorimotor territory of the striatum in monkey. J Comp Neurol 344:1-19.

Smith Y, Bevan MD, Shink E, Bolam JP (1998) Microcircuitry of the direct and indirect pathways of the basal ganglia. Neuroscience 86:353-387.

Smith Y, Raju DV, Pare JF, Sidibé M (2004) The thalamostriatal system: a highly specific network of the basal ganglia circuitry. Trends Neurosci 27:520-527.

Smith Y, Villalba RM, Raju DV (2009a) Striatal spine plasticity in Parkinson's disease: pathological or not? Parkinsonism Relat Disord 15 [Suppl 3]:S156-S161.

Smith Y, Raju D, Nanda B, Pare JF, Galvan A, Wichmann T (2009b) The thalamostriatal systems: anatomical and functional organization in normal and parkinsonian states. Brain Res Bull 78:60-68.

Somogyi P, Bolam JP, Smith AD (1981) Monosynaptic cortical input and local axon collaterals of identified striatonigral neurons. A light and electron microscopic study using the Golgi-peroxidase transport-degeneration procedure. J Comp Neurol 195:567-584.

Surmeier DJ, Ding J, Day M, Wang Z, Shen W (2007) D1 and D2 dopaminereceptor modulation of striatal glutamatergic signaling in striatal medium spiny neurons. Trends Neurosci 30:228-235.

Valjent E, Bertran-Gonzalez J, Hervé D, Fisone G, Girault JA (2009) Looking BAC at striatal signaling: cell-specific analysis in new transgenic mice. Trends Neurosci 32:538-547.

Vandermaelen CP, Kitai ST (1980) Intracellular analysis of synaptic potentials in rat neostriatum following stimulation of the cerebral cortex, thalamus, and substantia nigra. Brain Res Bull 5:725-733.

Xu ZC, Wilson CJ, Emson PC (1989) Restoration of the corticostriatal projection in rat neostriatal grafts: electron microscopic analysis. Neuroscience 29:539-550.

Xu ZC, Wilson CJ, Emson PC (1991) Restoration of thalamostriatal projections in rat neostriatal grafts: an electron microscopic analysis. J Comp Neurol 303:22-34. 